\title{
Client's Temporal Trajectory in Child Protection: Piecing Information Together in a Client Information System
}

\author{
Saila Huuskonen and Pertti Vakkari \\ School of Information Sciences, \\ 33014 University of Tampere, Finland \\ \{saila.huuskonen, pertti.vakkari\}@uta.fi
}

\begin{abstract}
Our study focuses on the information needed for overviews by social workers in child protection, and how information is presented in client information systems (CIS). Data consists of semi-structured interviews and social workers' observations while they used CIS in their daily work. The analysis was structured by the concept of temporal trajectory. We identified three major interconnected information strands: concern, child's private life and institutional actions. Their temporal lengths and aspects are disparate. CIS offers modest temporal overviews of clients' cases. Representing information strands as timelines on the interface would provide better overviews.
\end{abstract}

Keywords: client information system, child protection, temporality, work tasks.

\section{Introduction}

Child protection is a part of statutory social work. Its objective is to support children and their families in problematic life situations. Primarily work is done by offering in-home services. In child protection services, a client information system (CIS) is used to support social workers' work. It has a crucial role conveying information about a client's case. Information is recorded and used in CIS during a whole client process as part of daily work tasks [8]. The representation of the recorded information as a case overview in CIS is in the focus of our study.

Information is collected mostly in face to face interaction with different parties, and it is afterwards selectively recorded in CIS. Information in CIS can be plentiful especially in long and active client processes. It gets demanding to piece together an information puzzle to form an extensive overview of a case. Thus, fast and effective information representation in CIS would be essential so that social workers could rapidly make sense of how a client's case has evolved over a period of time. The overview available at-a-glance has value in several work tasks [8]. This is the case, for example when a new worker takes over a case or an old timer with heavy case loads tries to summon up a memory of what the client's story is. Obviously, the effective overview in CIS constitutes two intertwined things: the essential information that is well documented and CIS' ability to present it in a concise manner. 
Social workers' work orientation is holistic. This means understanding a person as a whole and as a part of social context [9]. The work requires a wide perspective about a client situation. However, the current systems do not seem to offer appropriate tools to present a client's situation fully. Systems are criticized, splitting holistic case information into pieces [7], [16], [33] and not fulfilling the task they were originally meant for [3]. Thus, a misfit between the needs of social workers and the way CIS present information seems to be obvious.

Despite the strong criticism there is a lack of studies, which analyze social workers' perception of holistic information needed in their work tasks, and how CIS supports representing and using this information. By exploring in detail what kind of overviews of client cases are needed we can create results for developing CIS to better fit to social workers' tasks.

We approach the child protection information from the temporal perspective. The importance of temporal perspective in research has been recognized in information studies [24], [26], social work research [19], [31] and CSCW/HCI [20]. Our research is situated at the intersection of these three disciplines.

Our research has three objectives. Firstly, we examine what kind of information according to social workers belongs to a comprehensive temporal overview of a client case in child protection's in-home services. Secondly, we give an account of how this information is currently presented in CIS in our research sites. Thirdly, we relate a need for 'overviewable' temporal information to work tasks. Temporal trajectory [20] [28] is used as an analytical concept. This concept offers an analytical lens to look at social workers' work with a client's case in temporal context.

Our study is based on field work realized in in-home services in child protection in Finland. In-home services are offered to a child and his family based on child welfare assessment. The services are defined by legislation. The field work consists of social workers' interviews and observation in real working situations with CIS.

The paper is structured as follows. In the first section, we describe the conceptual framework and review the previous literature. Next, we present the research setting including the research site, data collection and analysis methods. Then we introduce the results starting by reviewing social workers' perception of information that belongs to a client's temporal trajectory. Then, we analyze how the information with temporal perspective is presented in the CIS of our research sites. The last part of the result section looks at a temporal trajectory and CIS as a part of social workers' work tasks. We conclude with binding our three research objectives together, and outlining some design ideas.

\section{Conceptualization of Research}

Time and temporality both have a central place in social work practice. Many social work activities, the notion of intervention and change and social workers' sequential narrative accounts of cases have an aspect of temporality [31].

Temporality is embedded in different types of processes in social work. The processes have been defined from the perspective of work as the series of encounters and activities during the professional services [5]. The processes can be approached from the perspective of an individual client, too. Then a process is seen through 
human growth and changing circumstances [9]. The concept of trajectory originates from the studies that described work in medical settings. The central idea of the trajectory was to depict the organization of work associated with the course of the illness of a patient [28].The generalized concept of trajectory is understood as the evolvement of a phenomenon over time plus action, interaction between multiple actors and unanticipated contingencies related to phenomenon [27].

A further developed concept, temporal trajectory, is defined as a structured timeline that consists of activities, events and occurrences over time. The temporal orientation covers the past, present and expectation of future activities. This wide perspective makes it possible to see the relationship of activities and to take a look at patterns of former activities and anticipate the progress of a case. [20]

The temporal trajectory embodies similar elements as narrative accounts, traditionally valued in social work. Narrative has thematical development; it shows causality and it tells the story with a beginning, middle and end [1] just like temporal trajectory presents evolvement of a case over time.

We complement our analysis with three aspects of temporality: episode, interval and eon. Episode means a short period of time; interval a long period of time with distinct starting and ending; eon is a long continuous period of time [26]. The aspects are used to give a temporal shape to information in the trajectory.

\section{Related Research}

The core critical arguments in social work towards CIS concern the way of presenting information. The CIS has been seen as a threat to holistic and narrative information [16]. Reasons for the misfit might be that information is forced into a certain format [22] with too much focus on facts [14] and by this way being irrelevant to practitioners.

Recently, there have been plenty of studies around the British Every Child Matters Reform and its technological solutions. The main result of the studies is that this new technology does not optimally support the work practice. Integrated Children System does not offer a complete picture of a client's case because of a lack of contextualized narrative [33] and a lack of a common family file [7]. The research realized more than ten years earlier among Norwegian social workers ended up with similar results: it was impossible to pull together the full client history to view it at a quick glance since information was as pieces on the computer screen [9].

Physicians shared similar problems in gaining an overview from electronic heath records. Navigation between different screens lost the overview [2]. The studies seem to indicate that different professions share similar needs and similar problems.

Studies on reading the case files indicate that there is need for fast access to information. In CIS information is skimmed through with the aim of locating the most essential parts of it. Rapid access for information representing case overviews was valued, in particular. [8] The similar type of skim reading was recognized already in the time of paper documents both in social work [12] and in the medical context [15].

A structural client's assessment form (CAF), also part of the British Reform, splits information into fragments [25], disrupts the temporal and narrative display of 
information [32] and limits the expression of concern by highlighting a more robust assessment of needs [7]. The CAF form was used more in parts that offered possibilities to produce traditional narrative information [17]. Findings are alike in the study realized in Israel in the juvenile home context, where the structured assessment form was considered cumbersome and incapable to capture the client's unique information [23].

The temporal aspect of information and its implications for system design have been under study in computer supported co-operative work. The studies have been realized in health care. Health care staff valued seeing information organized chronologically because in that way they were able to situate the patient within the temporal trajectory and gain better understanding of a current situation [20]. The need for retrospective or prospective information depends on the work task at hand [21]. The study concerning chronic patients' illness trajectories highlighted the value of a good overview of a patient and as well an evolvement of medical information over time [13].

The previous studies point out a need for a chronological, time-wise approach to structure information, and the need for an overview of a client's situation available at a glance.

\section{Research Design}

\subsection{Research Questions}

We are interested in a triangle that constitutes information, its temporal presentation in CIS and how they intertwine in social workers' work tasks in child protection. The precise research questions are:

- What information strands belong to a client's temporal trajectory as perceived by social workers?

- How is a temporal trajectory presented in CIS in our research sites?

- How do social workers manage a temporal trajectory in CIS?

\subsection{Study Environment}

The field work took place in three Finnish municipal social service organizations offering in-home services in child protection. The in-home services defined by the Child Welfare Act (417/2007) include, e.g. support persons and families, financial help and family workers' visits at home. The data was collected in the year 2008.

In city $\mathrm{A}$, the number of front line social workers in child protection in-home services was ten, in city B nine and in city C 35. Outside of office hours an emergency department was responsible for urgent situations. Staff turnover was relatively high in the research sites. Often several social workers took care of a client's matters over time.

A social worker was in charge of a varying number of children. Most of the time a co-worker was also named for a case. Among interviewed social workers the caseload 
was heavy, varying from about thirty up to over a hundred cases. Social workers' task was to manage a client's case, e.g. by assessing need for the services, keeping in touch with a child and his/her family and other parties involved in a case, and organizing services. The social work managers occasionally took part in client work. They gave consultancy to their subordinates, carried out some official decisions and reacted to clients' contacts.

CIS had an integral role in managing clients' cases. Each child has their own file in CIS (Fig. 1). The current CIS was introduced to our research sites A and C in 2004 and in site B in 2006. Previously, another system was in use. In the implementation stage, only the basic information of active clients was manually entered to current CIS. Because of the lack of automatic conversion, narrative case reports were, however, left in former CIS. Each research site tailored CIS to match the local needs.

CIS has separate modules for documentation, structural family information, decision making, scheduling and statistical presentation of work activities. In the documentation module, social workers keep up the chronological case report and write a structural client plan. Family module depicts the people living in the same household. Decision making module is used, for example, when services are granted for a client. Scheduling module presents the forthcoming appointments. Work done module is used to numerically describe the past actions. Each module can be purchased separately. Therefore, there is a slight variation in what modules are available across research sites.

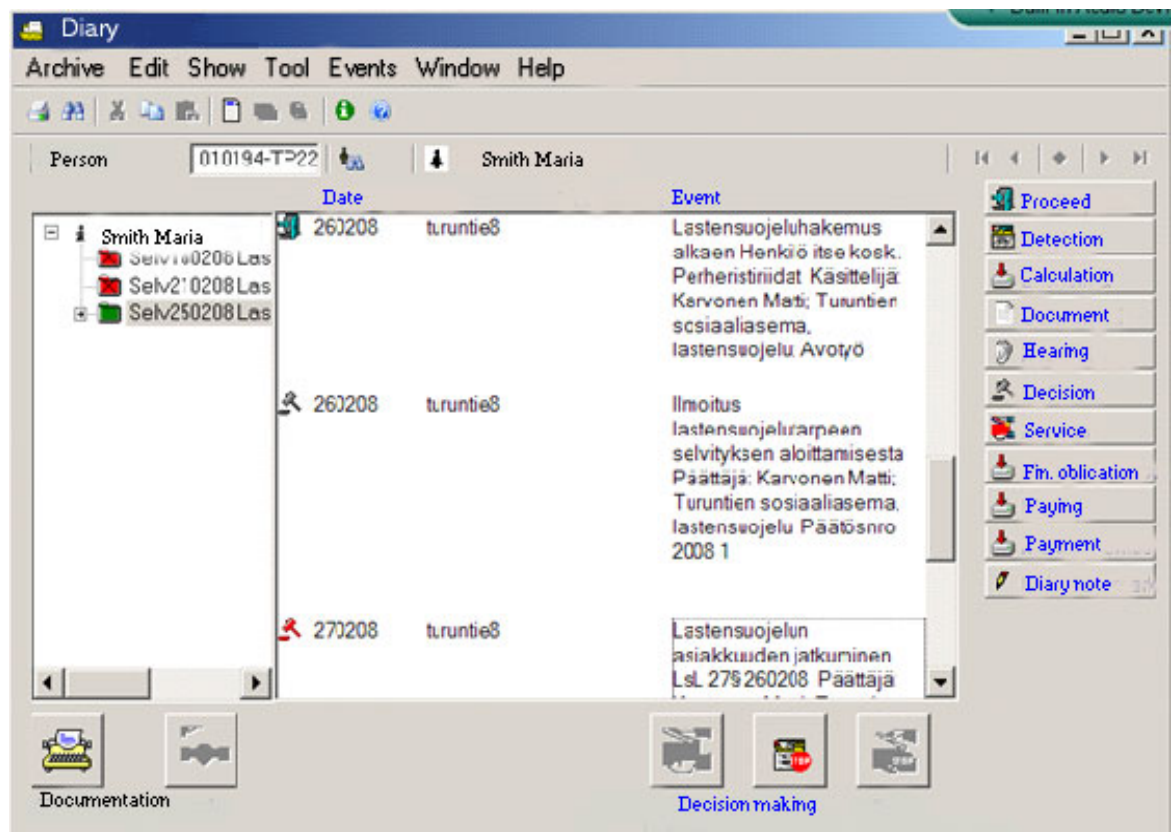

Fig. 1. The diary view of CIS - translated in English (fictional client) 
The so called diary view (Fig. 1) of a client serves as an access point to different modules. There is no possibility to navigate directly from one module to another but a loop through diary view is needed. The navigation buttons to the modules can be seen on the right side of the screen capture. The diary view also shows different files (on the left) and offers a modest glance at recent decisions of a case (on the middle of the screen). CIS offers limited search tools for the social workers. It is possible to search clients by their name or ID. However, there is no search option within a case report.

\subsection{Data Collection}

The study employed semi-structured interviews and field observations. The interviews were carried out first. Willingness to participate in observations was asked during the interviews and the actual observations were realized later. In city A and B the majority of social workers were interviewed whereas in city $\mathrm{C}$ about one fourth of the workers participated. The interviewed social workers had varying work histories in child protection services. There were novices, those who had a couple of years work history and a few having a long term career. The observed social workers had a heterogeneous history of using CIS. Some had experience only from this particular CIS and some had used various types of systems previously.

Before the actual interviews, a pilot interview was conducted to check the functionality of the questions and time required for an interview. Our data consists of 33 semi-structured interviews with social workers (23), social work managers (7) and system administrators (3). The interviews took place at the offices of the social workers, with only one exception at the university. The core themes of the interviews were work related to child protection, documentation and use of CIS as part of work. In sixteen interviews, social workers described an individual client's case by drawing a timeline and network map about persons involved in a case. The cases had a role of a critical incidence [29] allowing more focused and concrete questions. The total interview data is about 62 hours. One interview is about two hours in length. The interviews were tape-recorded and fully transcribed.

Twelve field observation sessions were realized in real-life settings. Eleven social workers were observed, one social worker twice. The social workers have a practice to book up time to do documentation and other work with CIS. The social workers were asked if it was possible to join those sessions and follow their work. The social workers worked normally in these observation sessions. The only exception was that based on their own choice they explained and reflected about their actions concurrently every now and then. This way, a verbal protocol [11] was produced. Observation data consists of hand written field notes from all sessions and the taperecorded commentary by the social workers from ten sessions. In two sessions it was not appropriate to do recording. Field notes were written up shortly after the observation session. Later on, the commentary of the social workers was transcribed word for word. The observation data is about 40 hours. The sessions varied from about two hours to the whole working day.

\subsection{Data Analysis}

Firstly, the interviews were read through several times to become familiar with the data. After that, appropriate parts of the data were collected under four wide themes 
according to research questions. The data extractions were thoroughly scrutinized and summarized with few words in the tables. This enabled us to analyze the dimensions of phenomena in a more itemized way.

Each critical incidence - client case used as an example in the interviews - was pulled together so that it formed a consistent account. This meant that all events were listed chronologically. Also institutional actors and people related to clients' private life were listed. The use of information and CIS was identified and itemized in the critical incidents.

From the interviews, those of the front line social workers' and social work managers' were used as a primary source. The interviews of systems managers offered background information to understand the properties of CIS.

The notes from the observations and social workers' commentary during the sessions were combined. Thereafter, the data was organized according to functional entities. A functional entity is a single task in CIS consisting of a sequence of actions occurring in order to reach a certain goal. The functional entities include activities like writing a case report and making different types of decisions. These functional entities were studied more carefully from two aspects: how social workers keep up the temporal trajectory and how they look for a temporal overview of a case.

The different datasets shed a different kind of light on the research questions. The interviews offered an overall picture of significant information and a role of CIS as part of work. The critical incidences within the interviews gave a shape to the clients' trajectories. Observations and social workers' verbal protocol concretized the humancomputer interaction.

We take two standpoints to temporality when we present the results. Firstly, we describe the temporal trajectory in general. Secondly, we link temporal aspects (episode, interval, eon) [26] to information presented in the trajectory.

\section{Results}

First, we describe information that the social workers consider as important for understanding a client's trajectory. Next, we analyze how this information is presented in CIS of our research sites, and the capability of CIS to give a temporal overview of a client case. Lastly, we briefly discuss social workers' strategies of producing and using the temporal trajectory in CIS as part of their daily work.

\subsection{Information Strands in a Temporal Trajectory of a Case}

Social workers clearly highlighted the importance of seeing and interpreting a client's situation as a temporal continuum. This meant a chronological and consistent narrative that could depict what had been the starting point and what had happened during a child protection process. The need to observe changes in the process was expressed by a social worker: "What has changed, if there is something new. I aim to depict the trajectory." (O 1)

However, it turned out to be more difficult for the social workers to define what kind of information belongs to the temporal trajectory. It is not about working around a single issue but rather handling a case from a much broader perspective. As 
expressed by one of them: "We manage a person's life in its entirety so what would not be meaningful." (3) A full trajectory is constructed from several information elements. Trajectory management is reminiscent of piecing a jigsaw puzzle together. The core of the problem is:"Also those small pieces of information are important so it is not enough to put down summarily those big outlines." (1)

The client's records serve as a temporal boundary object. Information is recorded in a specific moment and it might be used months, even years later. Often, however it is unclear what information is needed in the future. Besides the wide working orientation, situational and temporal factors also seem to somewhat explain what is considered as relevant information.

Three major information strands of the temporal trajectory were identified. They were strands of concern, a client's private life and institutional actions. They were intertwined. To understand a client case as a whole requires seeing the strands together.

Concern Strand. To the social workers, the concern strand explained the varied reasons for the beginning and continuation of customership in child protection services. The social workers appreciated concrete expressions of concerns. The essential was to find out: what is the concern about? The list of major reasons for concern was relatively limited. Moreover, often it is not possible to articulate well what it is all about.

It is common that the concern strand has several actors expressing their point of view about a client's situation. In some cases, opinions about concern can be contradictory, and also contradict the social worker's opinion.

The temporal strand of concern is not necessarily linear, stretching over the past and the future, as the examples told by social workers demonstrated. The arc can be cyclic when there are passive and active stages of concern. The concern can fluctuate when a level of concern takes turns. The course of concern might be steady or cumulative with increasing issues of concern coming out. The concern strand can consist of several of these features.

Concern is hard to fit into any of the temporal aspects (episode, interval, eon). It is rather an all over state continuing across the temporal trajectory. In that sense, it belongs to the temporal aspect called eon. However, the level of concern can have peaks or periods of good or bad times. In those situations, the episode and interval aspects of time are applicable.

Private Life Strand. The essential information in the private life strand concerns the whole family, not only the child. We recognized three major information themes that social workers talked about: major events, everyday life at home, and family as a network. Noteworthy is that information is often needed about the past events and life situation from the time before the child protection. After all, the private strand describes "the history of family".

All the aspects of time match up with the private strand: episodes for events, interval for certain periods in family life and eon to depict continuity in everyday life.

Institutional Strand. The reason and level of concern determine what kinds of services are organized for a child and family, and what institutional actions are taken. The institutional strand describes the work done in the child protection. The social workers seemed to need a long term view of services: what had been in use but 
terminated, how did services get started, what currently is going on, how long were the service periods, and what are the plans for the future. The evaluative information about the impact of services and family attitudes towards services were needed as well. The analysis of the network maps of the critical incidents revealed that social workers co-operate with a multitude of people. There were co-workers within their own social service organization (emergency department, adult services, welfare for intoxicants) and outside of it. All of them are potential information producers.

From the temporal aspects, episode and interval seem to felicitously depict the institutional work.

To conclude this chapter, we use data extracts to demonstrate the temporality and a contingencies, in the work.

"Q: What is the essential information in child protection, what should be available from each client?

A: Well, why the customership exists and what is the goal and what is the plan to reach the goal.

Q: Hmm.

A: Often we are reproached that we don't have any plans in our work. We are making plans today and in the next day our plans might be vitiated because of a single phone call.” (6)

We can summarize the core findings of information strands in a temporal trajectory to the following: reason and level of concern; services and actions child protection has taken plus their impact; major events, everyday life, and a child's network are core information needed in the overview to support social workers in their work. The intertwined strands have different temporal aspects and lengths, the institutional trajectory maybe being the simplest one to represent. In the next chapter, we take a closer look at how CIS is capable of presenting the three identified strands as a trajectory.

\subsection{Presentation of Temporal Trajectory in CIS}

We mirror information presentation in CIS through a specific case of Maria. The temporal trajectory (in italic in the next paragraph) is the idealization of several critical incidences presented in the interviews. The strands of the temporal trajectory concern, private life and institutional - are narrated side by side.

Maria's customership was opened at the beginning of 2005. The school contacted child protection. Maria attended school irregularly and looked unbalanced. Her parents were divorced. She lived with her mother and two younger siblings. The social worker called the teacher and the mother. There were meetings with Maria and the mother at the office and a visit to home. The social worker noticed that the mother seemed to have health problems, balanced with short term jobs with no time and energy for children. The social worker organized a support family (obtained after six months waiting) and financial support for Maria's hobbies. The mother's partner moved in and out, the family moved within the city, Maria participated in a summer camp. Everyday life rolled on. In 2006, a new social worker took care of Maria's case. Meetings were irregular. At the beginning of 2007, the neighbours made two child welfare notifications: children had been alone at home. The third social worker 
visited the family. She could not figure out the situation, just made observations about home and the mother's tiredness. To strengthen the support, a family worker started to visit the family. However, the work was soon discontinued because the mother wanted it to be. In 2008, customerhsip was on hold. Then the social worker got a very serious child welfare notification by anonymous phone call - it was time to react.

Next we take a look at how information about Maria's trajectory is distributed, and can be found within CIS: in modules of documentation (includes e. g. case report, client plan), of family, of decision making, of scheduling and of work done.

Concern Strand. There are four child welfare notifications. The first one is from the school, two later ones from the neighbours and the last anonymous one. The other form of concern is that of the social workers: the observations about the mother's tiredness in the first place. Later it is only a hunch when the social worker is not able to clarify a crux of the matter. The notifications have their own registry in CIS, where they are recorded. This information is brief and structured: a date, the name of informant and the content of contact. In most cases, the social workers also write as a link a short entry about the notifications to the case report. The social workers' own concern and assessment of its level is embedded in the case report. The worry expressed by different actors during discussions is also in the case report.

Private Life Strand. There are few events listed in Maria's and her family's life: a summer camp, changes in the mother's relationship and a change of residence. We can also find references to the time before customership. In the past the parents have divorced. There is also information about everyday life. These are reported on the case report. In addition, the official changes in the household composition (move of the mother's partner) and a move during the customership are depicted in structured form in the family module.

Institutional Strand. During the course of the trajectory there were meetings, phone calls and home visits. Besides events, the social workers made four formal decisions. The first one is about to start the customership and three latter ones about the services: financial support, a support family and family work. The staff turnover is also a part of institutional strand: three different social workers took care of Maria's case. The institutional work is fully reported on the case report in the documentation module. The entry of a short event starts with the date, social worker's name, the name of event and the listing of participants. Thereafter, the content of the event and discussions are described. It is a practice to mention the decisions made in the case report. The institutional work is monitored in three other modules. In the decision module are the structured decisions including written justification. The work done module indicates the already realized actions (phone calls and meetings etc.). It briefly depicts the type of action, the number of participants and time in minutes spent with a work task. The scheduling module orientates to the future. It indicates the forthcoming meetings and home visits. These modules, however, give only a limited view of the work.

Obviously, the case report on the documentation module offers the most comprehensive account of Maria's case. It binds all three information strands together. Its temporal perspective covers the time before child protection, current time and the future. The information in the other modules is mostly used for administrational and accountability purposes. Their timeframe covers only the current 
time in the child protection. The information presentation is multifold since the same information can be found in different modules expressed in a different form. The information strands, modules containing information about them, and their temporal aspects in CIS are summarized in Table 1.

Table 1. Information strands, modules and temporal aspects in CIS

\begin{tabular}{|c|l|l|}
\hline Strand & \multicolumn{1}{|c|}{ Module } & \multicolumn{1}{c|}{ Temporality } \\
\hline Concern & $\begin{array}{l}\text { Documentation } \\
\text { Notification registry }\end{array}$ & $\begin{array}{l}\text { episode, interval, eon - past, current, future } \\
\text { episode - current }\end{array}$ \\
\hline Private life & $\begin{array}{l}\text { Documentation } \\
\text { Family module }\end{array}$ & $\begin{array}{l}\text { episode, interval, eon - past, current, future } \\
\text { episode - current }\end{array}$ \\
\hline Institutional & $\begin{array}{l}\text { Documentation } \\
\text { Decision module } \\
\text { Work done module } \\
\text { Scheduling }\end{array}$ & $\begin{array}{l}\text { episode, interval, eon - past, current, future } \\
\text { episode, interval - current } \\
\text { episode - current } \\
\text { episode - current (orientation to future) }\end{array}$ \\
\hline
\end{tabular}

According to the social workers the fastest way to get an overview about a client's trajectory was to read through a client plan. It summarizes the history of the family and clarifies the client process and goals set. In the client plan information is ready selected and analyzed. However, not all clients had one. In those cases, the social workers were dependent on a case report if they wanted to figure out a child's history as a whole.

Overall, the significant issue was the difficulty of identifying the thread running through the case report: finding the course of action. This was particularly the case in active client processes when reports could be "thick like a brick". The social workers noted that the concern, major events, goals and their realization did not come up clearly enough. The case report contained those small pieces of information recorded just in case. The other reason is that the history is made up of different entries as life unfolds.

CIS could give only superficial support depicting an overview of the case. The diary view visualizes only the different files and major decisions. The following data extract exemplifies not only how information comes up in the case report but also the social worker's fast interpretive skills. She discusses information through institutional strand: numerous entries are a message from active working that likely indicates the seriousness of a case. By looking at the time frame she analyzes the shape and stages of a trajectory. The work of the emergency department is a message about possible crisis and contingencies.

"You can at a glance see how many entries there are [in a case report]. Sometimes it scrolls a long time to get open. Then you know that there is plenty of writing and a long customerhip. You can take a look at what time frame those entries have been made...You can see from the initials if it has been the emergency department working over the case. You can make those short observations." (4) 
Client information was spread over several modules in CIS. Information of institutional strand was in four modules, and concern and private life strands in two modules. In the documentation module, case report compiled information of all strands. However, it was difficult to get an overview about a client's case based on it.

\subsection{Interacting with CIS: Managing and Using a Temporal Trajectory}

Social workers had different strategies of managing and using trajectories when they interacted with CIS. Since the major source of information was the case report, the majority of the management work was done there. The social workers' goals were first of all, to keep the trajectory as a whole in the case report by referring to possible information in other modules. Secondly, there were different types of attempts to point at the essential information in the case report. To gain a temporal overview of a case, social workers approached the case report either by skimming text through or by reading intensively.

Managing Trajectory. We identified three tactics to keep up the temporal continuum in the case report. Firstly, the social workers created links to information outside of the case report by writing short references. They indicated whether there was more information in another document (e. g. in the client plan) within the documentation module or in another module. In the latter case, references were mostly made to decisions that existed in the decision module. The social workers also created traces to hint that more information could be found from the family members', siblings' or parents' files in CIS. In the client cases that had started before the implementation of CIS, there were occasionally references to old CIS. Often, the social workers made a note if there was more information in paper files, too. Secondly, the social workers wrote, although very rarely, summaries. The summaries related to the past, a time before a child became a customer in this particular organization. This was the case, for instance, when a child had a history in another municipal's child protection service or there were previous remarks concerning the family. Occasionally, summaries were made about paper reports. Thirdly, in some situations the social workers kept the case report consistent by keeping up a dialogue between past and current entries and between the entries of co-workers. The current entries were reactions to the past ones. The linking strategy (manually added notes to the case report) was relatively simple and more technical by nature, instead summary writing is rather a writing practice.

Indicating the essential information was realized by adding titles to structure the text, using bold fonts or making listings to the end of entries. The bold fonts were used, for example, to highlight turning points or serious events. Serious issues that were raised for discussion for the first time could be entered in bold text. The social workers used listings in the entries, mostly to summarize agreements and actions to be taken in the future. These tactics aimed at offering handles for fast access to information.

Using Trajectory. We identified two tactics, holistic check up and intensive reading, as the social workers' ways of familiarizing themselves with the whole trajectory in CIS. The holistic check up means skimming through the text in the case report and trying to locate the essential parts of it. In the intensive reading the social workers 
carefully go through the entries. In some cases, they print out the case report and rather read this printed document.

The time available to read the entries, the familiarity of a client, a work task or a work situation at hand were some of the reasons that dictated the ways of reading. The social workers skimmed through the past entries before the meetings, when they were on the phone with a client or when they evaluated the continuity of a client's case quickly. The previous information had a special significance to the social workers who took over an old case and tried to figure out the past. The taken into care decision or making a first client plan for a long-term customership required intensive reading.

We illustrate these two information use tactics by presenting two functional entities from the observation sessions. On the tables, the left column describes the major moves made in CIS. The right column includes the essentials of the verbal protocol. The fist example depicts the holistic check up and the second one intensive reading.

In the first example (Table 2), the social worker goes through a client's list in a semi-annual customers' 'check-up day'. She evaluates whether the customership needs to be sustained or be terminated. She navigates from the child's file to the mother's file, returns to the child's file to close down the case and finally closes down the sibling's case too. She looks at information from the temporal perspective. Firstly, she looked at the starting time, and then stages in the client process. She notices that the situation is passive. She uses all information strands of the trajectory. She makes observations about the services that had been in use (institutional strand), reflects the concern and finally seeks consolidation from the mother's income support file (private life strand). She remembers the mother from engagements at the office. The information in CIS combined with her own experiences support decision making. She decides to close the case and the sibling's case as well. The task does not require much time.

Table 2. Holistic check up - evaluating the customership

\begin{tabular}{|l|l|}
\hline Moves in CIS & Verbal protocol \\
\hline 1. skims the case report of the child & "This has happened in 2004. This case \\
2. skims the case report of the mother & is still open. There has been family work \\
3. checks the income support & but terminated. Well, in 2006 - now I \\
calculation of the mother & got hang on this. They have been in a \\
4. returns to the child's file and & summer camp. I remember that the \\
terminates the customerhip & mother has been here for income issues. \\
5. moves to the sibling's file and & The past action was two years ago. I \\
terminates the customerhip & $\begin{array}{l}\text { don't have any child protection work } \\
\text { here... I take a look by mother's } \\
\end{array}$ \\
& $\begin{array}{l}\text { name...There is neither work going on } \\
\text { nor is there concern. " }\end{array}$ \\
\hline
\end{tabular}

Our second example (Table 3) draws from the situation in which the social worker prepares a tentative client plan to be discussed in a meeting. The child has the history of several years in the child protection. The social worker has been in charge of the child's matters for about a year. According to her, the customerhip is activated 
because of a crisis. For the plan, she must piece together a compiled package about concerns, private life and institutional actions. She starts by glimpsing a case report and correcting some spelling mistakes in it. Thereafter, she prints out the case report's entries from the past year. Then she moves to the actual task at hand: preparing the client plan. She reads the case report both from the CIS screen and from print. She tries to figure out the course of action. She is lost in the text: she needs to locate information piece by piece. As she says, information is fragmented in the case report. She makes notions about services (institutional strand), child welfare notifications (concern strand) and family situation (private life strand). She finds out that the situations of the same kind reoccur and continue in the family's life. That is an important piece of information. She copies and pastes between the case report and the client plan. She spends most of the afternoon familiarizing herself with the client's case. To boot, there would be even more information in old CIS and paper files.

Table 3. Intensive reading - preparing a client plan

\begin{tabular}{|l|l|}
\hline Moves in CIS & Verbal protocol \\
\hline 1. skims the case report of the child & "I need to ask if somebody can take a \\
2. checks assistance (how to check & look at information in old CIS. \\
spelling in CIS) from a colleague & You just have to search for the \\
3. prints out the case report from the & information. If a case report is all you \\
past year & got you are totally lost. \\
4. works with a client plan & This is quite a patchwork. \\
5. reads the case report carefully & If only there could have been a way to \\
6. copy-pastes between these two & pick up [the services] somehow. By now, \\
documents & I have spent almost 1 1/2 hours on this. \\
7. prints out the half-done plan & I could take a look at the paper files too. \\
& What had happened in the child's life, \\
& what has it been, the background \\
information is laborious to find. The & information is fragmented there." \\
\hline
\end{tabular}

The two previous examples depict the role on information and CIS as part of two different types of work task. The social workers interact with information and CIS alike. In both cases, the social workers search information from the perspectives of three information strands. They also make notions about the past and the current situation of a case when trying to assess the possible future of a child. The tasks require both the identification of essential information and navigation between different modules in CIS. Apparently, CIS could offer more support to realize the tasks. However, it is a matter of the social worker's professionalism, too. As expressed by the social worker when talking about whether there was enough information for preparing a taken into care decision: "Yes there was, but you have to see the wholeness. And [understand] what to do with that information." (7) 


\section{Discussion and Concluding Remarks}

Summarizing the Findings. In child protection, there is a need for case overviews presented by CIS [8]. The overview would support the social workers' work that is framed by limited time resources, heavy caseloads and situations when immediate reactions are needed. We approached the construction of the overview from three perspectives. Firstly, we identified the core information strands belonging to the case overview. Secondly, we analyzed how these information strands were presented in one particular CIS. Thirdly, we observed how the social workers managed and used these strands in CIS.

In the previous studies, CIS has been considered as a threat to presenting holistic information in social work [7], [16], [33]. Holistic information has been taken for granted with no exact explanation of what it is and how it should be presented in CIS. To design an overview, however, its core information elements should be defined. We identified three interconnected information strands in a client's trajectory: of concern, of private life and of institutional actions. The social workers preferred to see the strands from a temporal perspective. They needed information about the past, the present and the planned future of a child. The temporal continuum also describes change, continuity and permanency. Visibility of these three factors is central when trying to understand the complexity of case work [19].

The information elements of the concern and private life strands included three temporal aspects: episode, interval and not so clearly time sliced aspect, eon. Instead, information elements in the institutional strand included the aspects of episode and interval. Consequently, the information in the institutional strand is easier to describe with exact time stamps than in the two other strands. Time has been modeled according to instant (aka episode) and interval in information system design [4]. Our findings indicate that information can be temporally depicted similarly in CIS in social work.

Information was distributed across separate modules in the CIS analyzed. However, the case report had a central role to keep three information strands together. The report is a sequential account [31] but still, the entries can refer to the past, current and future issues in a child's life. Thus, it is not only the CIS fragmenting information as has often argued [7], [16], [33] but as well the nature of work that generates information as time sliced pieces. The social workers can only write the child's story as it unfolds. Information is obtained piecemeal during a client process.

The social workers managed a client's trajectory within a case report in two ways. Firstly, they created references to information existing in other modules or paper files. They did this to ensure a temporal continuum of a case at least in one place. Secondly, the social workers occasionally highlighted the essential information to create help in identifying the essentials. They used their own tactics to patch up the missing properties of CIS.

The social workers had to piece together an overview of a case in their minds. They either tried to scan quickly through a case report (holistic check up) or if necessary read through a case report carefully (intensive reading). The previous studies on medical [15] and social work [12] context identified skimming as one 
approach to documents. Obviously, exhaustive information is not always needed. Even a hint, e.g. bolding, can fill the information gap and guide in work task performance.

Implication for Design. There has been growing interest toward temporal representation of information. Proposals have been made to visualize information in timelines, e. g. with patient data [6], [30], personal histories (LifeLines) [18] and newspaper news [10]. Our findings are in line with these proposals. The social workers themselves directly referred to diagrams as alternative ways to depict the core history of a child. Also, assuring a temporal continuum of different documents is a way to keep a child's case history coherent.

Three information strands found could be depicted as parallel timelines. The construction of timelines requires collecting information in different CIS modules together. The timelines could have 'pointers' with snippets to actual documentation. The social workers could choose whether to draw on a summary or navigate to the actual documentation. These alternatives would support two reading tactics: skimming and intensive reading. An opportunity to mark possible contingencies [27] or turning points are similar to the social workers' currently used tactic to highlight issues in a case report.

The child welfare notifications are concrete expressions of concern to be compiled to the concern timeline. The challenging part, however, is to depict the social workers' own assessment or hunch of concern. As carefully proposed in a couple of the social workers' interviews, the concern could be 'measured' and indicated with colour codes: red for high and green for low concern level. It is a double-edged sword though. The coding might be supportive but it also adds to the work of social workers.

Private life constitutes events that are, at least partially, possible to collect to the timeline. Family is a central context of a child's private life. It includes siblings, parents, stepparents, grandparents etc. Depicting a family as a social network graph might ease the understanding, in some cases, of complex family structures. In many cases, the social workers navigate between family members' files. The network graph could offer access points between the files.

The timeline of institutional strand could depict given service periods and actions taken by social workers and other workers. This timeline would support seeing a cumulative arc of action on a trajectory [27] by showing all the provided services.

Creating a continuum of documents requires collecting entries from different modules and scanned paper statements to a 'document timeline'. Scanning possibility exists already now in CIS. Currently, the social workers sustain the continuum of documents in a case report by their linking practices.

System design should be done keeping in mind that social workers need a tool that is simple and easy enough to use [8]. The possible new features in CIS should not produce an extra burden for social workers.

Implication for Research. The construction of a timeline can be based on existing temporal markers in CIS. Taking a look inside at different documents, especially a case report, is essential. Thus, further analysis of temporal markers is needed. 
The social workers take a lot of effort to produce information about a child's case in CIS. Currently, CIS does not offer a coherent overview of a case that is available at a glance. It is possible to design a holistic overview of a case to match the social workers' needs and to support social workers' work.

Acknowledgments. This study is financed by the Academy of Finland grant 133053 . We thank the employees at our research sites for participation, and Tarja Pösö, Aino Ritala-Koskinen and Tarja Vierula in our CHILDINFO research group for helpful comments.

\section{References}

1. Aas, K.F.: From Narrative to Database: Technological Change and Penal Culture. Punishm. Soc. 6, 379-393 (2004)

2. Ash, J.S., Berg, M., Coiera, E.: Some Unintended Consequences of Information Technology in Health Care: The Nature of Patient Care Information System-Related Errors. J. Am. Med. Inform. Assoc. 11, 104-112 (2004)

3. Bell, M., Shaw, I.: Integrated Children's System Evaluation: Summary of Key Findings. DCSF-RBX-02-08 (2008)

4. Combi, C., Keravnou-Papiliou, E., Shaher, Y.: Temporal information systems in medicine. Springer, New York (2010)

5. Compton, B., Galaway, B., Cournoyer, B.: Social work processes, 7th edn. Thomson Brooks/Cole, Belmont (2005)

6. Drohan, B., Grinstein, G., Hughes, K.: Oncology Lifeline - A Timeline Tool for the Interdisciplinary Management of Breast Cancer Patients in a Surgical Clinic. In: Information Visualisation (IV), 14th International Conference, pp. 233-238 (2010)

7. Hall, C., Parton, N., Peckover, S., et al.: Child-Centric Information and Communication Technology (ICT) and the Fragmentation of Child Welfare Practice in England. J. Soc. Policy 39, 393-413 (2010)

8. Huuskonen, S., Vakkari, P.: Client Information System as an Everyday Information Tool in Child Protection Work. In: Proceeding of the Third Symposium on Information Interaction in Context, pp. 3-12 (2010)

9. Lie, M.: Technology and Gender Versus Technology and Work: Social Work and Computers. Acta Sociol. 40, 123-141 (1997)

10. Matthews, M., Tolchinsky, P., Blanco, R., et al.: Searching through Time in the New York Times. In: Proceedings of the Fourth Workshop on Human-Computer Interaction and Information Retrieval, pp. 41-44 (2010)

11. Miller, R.L., Brewer, J.D.: Verbal protocol analysis. In: Miller, R.L., Brewer, J.D. (eds.) The A-Z of Social Research. Sage, Thousand Oaks (2003), http://srmodev. ifactory.com/view/the-a-z-of-social-research/n117.xml

12. Monnickendam, M., Yaniv, H., Geva, N.: Practitioners and the Case Record - Patterns of use. Adm. Soc. Work 18, 73-87 (1994)

13. Munkvold, G., Ellingsen, G.: Common Information Spaces Along the Illness Trajectories of Chronic Patients. In: Bannon, L.J., et al. (eds.) ECSCW 2007: Proceedings of the 10th European Conference on Computer Supported Cooperative Work, pp. 291-310. Springer, London (2007)

14. Murphy, J.W., Pardeck, J.T.: Computerization and the Dehumanization of Social Services. Adm. Soc. Work 16, 61-72 (1992) 
15. Nygren, E., Henriksson, P.: Reading the Medical Record. I. Analysis of Physicians' Ways of Reading the Medical Record. Comput. Methods Programs Biomed. 39, 1-12 (1992)

16. Parton, N.: Challenges to Practice and Knowledge in Child Welfare Social Work: From the 'social' to the 'informational'? Child. Youth Serv. Rev. 31, 715-721 (2009)

17. Pithouse, A., Hall, C., Peckover, S., et al.: A Tale of Two CAFs: the Impact of the Electronic Common Assessment Framework. Br. J. Soc. Work 39, 599-612 (2009)

18. Plaisant, C., Mushlin, R.: An Information Architecture to Support the Visualization of Personal Histories. Inf. Process. Manage. 34, 581-597 (1998)

19. Pösö, T.: Revisiting Residential Care. Qual. Soc. Work 9, 27-42 (2010)

20. Reddy, M., Dourish, P., Pratt, W.: Temporality in Medical Work: Time also Matters. Comput. Supported Coop. Work 15, 29-53 (2006)

21. Reddy, M., Dourish, P., Pratt, W.: Coordinating Heterogeneous Work: Information and Representation in Medical Care. In: Prinz, W., et al. (eds.) ECSCW 2001: Proceedings of the Seventh European Conference on Computer Supported Cooperative Work, pp. 239258. Kluwer, Dordrecht (2001)

22. Sapey, B.: Social Work Tomorrow: Towards a Critical Understanding of Technology in Social Work. Br. J. Soc. Work 27, 803-814 (1997)

23. Savaya, R., Spiro, S.E., Waysman, M., et al.: Issues in the Development of a Computerized Clinical Information System for a Network of Juvenile Homes. Adm. Soc. Work 28, 63-79 (2004)

24. Savolainen, R.: Time as a Context of Information Seeking. Libr. Infor. Sci. Res. 28, 110127 (2006)

25. Shaw, I., Bell, M., Sinclair, I., et al.: An Exemplary Scheme? an Evaluation of the Integrated Children's System. Br. J. Soc. Work 39, 613-626 (2009)

26. Sonnenwald, D.H., Iivonen, M.: An Integrated Human Information Behavior Research Framework for Information Studies. Libr. Infor. Sci. Res. 21, 429-457 (1999)

27. Strauss, A.L.: Continual permutations of action. AldineTransaction, New Brunswick (2008)

28. Strauss, A.: Social organization of medical work. University of Chicago Press, Chicago (1985)

29. Urquhart, C., Light, A., Thomas, R., et al.: Critical Incident Technique and Explicitation Interviewing in Studies of Information Behavior. Libr. Infor. Sci. Res. 25, 63-88 (2003)

30. Wang, T.D., Wongsuphasawat, K., Plaisant, C., et al.: Visual Information Seeking in Multiple Electronic Health Records: Design Recommendations and a Process Model. In: Proceedings of the First ACM International Health Informatics Symposium, pp. 46-55 (2010)

31. White, S.: Time, Temporality and Child Welfare: Notes on the Materiality and Malleability of Time(s). Time Soc. 7, 55-74 (1998)

32. White, S., Hall, C., Peckover, S.: The Descriptive Tyranny of the Common Assessment Framework: Technologies of Categorization and Professional Practice in Child Welfare. Br. J. Soc. Work 39, 1197-1217 (2009)

33. White, S., Wastell, D., Broadhurst, K., et al.: When Policy o'erleaps itself: The 'tragic Tale' of the Integrated Children's System. Crit. Soc. Policy 30, 405-429 (2010) 